\title{
Motion-Induced Imbalance of Contact Impedance in ECG Capture: Comparison of Electrode Materials in Capacitive Coupling
}

\author{
Sebastian Pfeiffer ${ }^{1}$, Sebastian Meyer ${ }^{1}$, Oliver Amft ${ }^{2}$, Daisuke Anzai ${ }^{3}$, \\ Jianqing Wang ${ }^{3}$, Georg Fischer ${ }^{1}$, Jens Kirchner ${ }^{1}$ \\ ${ }^{1}$ Institute for Electronics Engineering, Friedrich-Alexander-University Erlangen-Nürnberg (FAU), Erlangen, Germany \\ ${ }^{2}$ Chair of Digital Health, Friedrich-Alexander-University Erlangen-Nürnberg (FAU), Erlangen, Germany \\ ${ }^{3}$ Graduate School of Engineering, Nagoya Institute of Technology, Nagoya, Japan
}

\begin{abstract}
Differences in contact impedance of the ECG measurement electrodes lead to asymmetries of the signal paths and thus result in reduced common-mode rejection and artifacts. Here, the imbalance of contact impedance is investigated for different types of electrodes with capacitive coupling in terms of static imbalance as well as dynamic variation during body movement. Flexible and incompressible materials like conductive foam and fabric showed the best overall performance. The negative effect of rigidity can partly be compensated by adding conducting foam, while soft materials can profit from an increase of electrode area.
\end{abstract}

\section{INTRODUCTION}

For the detection of sporadically occurring arrhythmiae such as atrial fibrillation, long-term monitoring of the electrocardiogram (ECG) is required. For that purpose, conventional Holter ECG devices provide a wearing period of 24 to $48 \mathrm{~h}$; longer wearing times of up to 14 days are afforded by recently proposed ECG patches [1]-[5], which were reported to exhibit an improved detection rate of cardiac diseases [6]-[8].

All of these devices, however, use electrolytic gels for galvanic coupling, which have a limited wearing time due to drying out and were indicated to cause skin irritations and even inflammation [9], [10]. To overcome these shortcomings, electrodes with capacitive coupling have been proposed, which dispense with direct skin contact (see, e.g., [11]). In particular, the authors have recently proposed a small wearable ECG device characterized by only passive components used between electrodes and instrumentation amplifier for the sake of increased symmetry between the signal paths and hence improved common mode rejection [12].

Aside from the signal paths within the electronic circuit, the differences in the contact impedances of the electrodes are a second source of asymmetries and open the door for interference. These electrode artifacts are well known in ECG applications, particularly in ambulatory use, and are mainly addressed by use of appropriate algorithms for detection and, as far as possible, compensation (see, e.g., [13], [14]). Here, we investigate this imbalance of contact impedances detached from further signal processing, quantify its effect during body movement and test whether it can be reduced by use of different electrode materials.

\section{Methods}

\section{A. Measurement System}

1) Electrodes: The following six types of electrodes were investigated, which couple the measurement system to the human skin. All of them had a circular shape and, if not stated otherwise, a diameter of $4.5 \mathrm{~cm}$.

Co Copper plate electrode realized as a printed circuit board. CoFo Same copper electrode as in 1 but combined with the conductive foam in Fo.

Fo Conductive foam 5770 made of polyurethane foam coated with copper and nickel, with a thickness of $3 \mathrm{~mm}$.

Mo1 Moss embroidered electrode manufactured by ZSK Stickmaschinen $\mathrm{GmbH}$, Krefeld. For embroidering, the pure silver coated yarn Statex Shieldex 33/10 dtex Z-turns with a resistivity of $<4 \mathrm{k} \Omega \mathrm{m}^{-1}$ was used.

Mo2 Same moss embroidered electrode as in Mo1 but with diameter $6.75 \mathrm{~cm}$, i. e., increased by $50 \%$.

Fa Conductive fabric electrode made of Shieldex Techniktex P130 (Statex, Bremen, Germany) with a thickness of $0.67 \mathrm{~mm}$. This fabric is made of a raw material of $78 \%$ polyamide and $22 \%$ elastomer, plated with $99 \%$ pure silver and coated with conductive silicone. To add stability, the very thin fabric was mounted on the rather rigid conductive foam E103-Hart provided by Schaumstofftechnik Nürnberg, Germany.

The copper electrode Co represents the direct realization of a capacitive coupling electrode as a PCB. A soft buffer from conductive foam is added to this stiff plate in $\mathrm{CoF}$, in order to increase the contact area. This conductive foam is investigated as a stand-alone electrode in Fo. A similar material structure is found in the electrodes made of conductive moss (Mo1 and Mo2); the different diameters allow to study the effect of electrode area on contact impedance. Finally, the fabric provides a thin and flexible material.

2) Measurement Setup: The electrodes were connected with electrode cables to a custom-made printed circuit board (PCB). This data acquisition circuit was designed according to [15] by use of Altium Designer V18.1.7. It is shown in Fig. 1. 


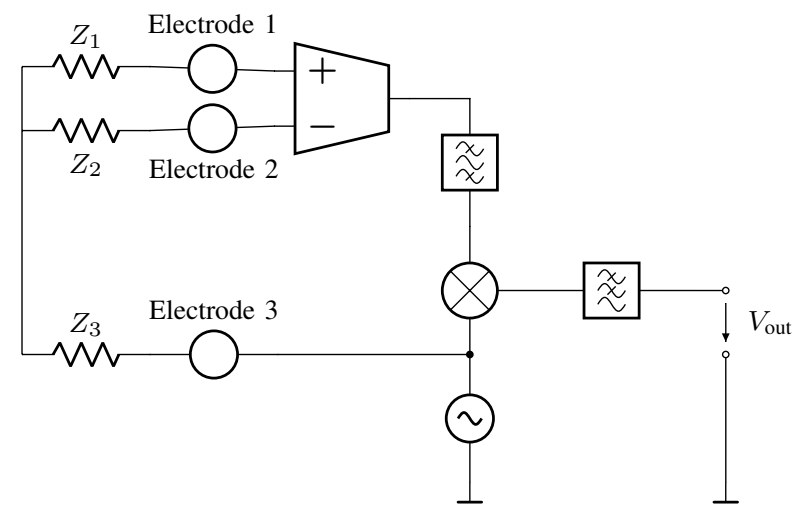

Fig. 1. Measurement circuit.

a) Sensor principle: Three electrodes are applied to the human body: The reference electrode no. 3 , in all cases a standard $\mathrm{Ag} / \mathrm{AgCl}$ electrode fixed to the abdomen, is used to feed a sinusoidal voltage $V_{\text {in }}$ into the body. Between the remaining two electrodes no. 1 and 2, made of the materials specified in II.A.1, the voltage is measured by use of an instrumentation amplifier (IA). Different contact impedances $Z_{1}$ and $Z_{2}$ of electrodes 1 and 2, respectively, lead to an asymmetric voltage drop over $Z_{1}$ and $Z_{2}$, hence to different input voltages to the IA and thus to a non-zero output $V_{\mathrm{IA} \text {,out }}$, which is proportional to the relative impedance mismatch. This impedance difference thus provides an amplitude modulating signal for the carrier signal provided by the voltage source. The demodulation is realized by a mixer and a subsequent low-pass filter.

b) Hardware realization: The $\mathrm{PCB}$ is powered by a conventional power bank with $5 \mathrm{~V} / 2.4 \mathrm{~A}$ output power. This is converted to $\pm 5 \mathrm{~V} / 250 \mathrm{~mA}$ dual-output power by use of the DC/DC converter TPS65133 (Texas Instruments, Dallas, Texas, USA).

The reference signal is generated on board by a Wien bridge oscillator. Its design is based on the low noise and low distortion operational amplifier LT1037 (Analog Devices, Norwood, Massachusetts, USA) and follows [16]. As instrumentation amplifier the INA 116 (Texas Instruments) was chosen due to its input resistance of $>10^{15} \Omega$ and a differential input capacitance of $<0.2 \mathrm{pF}$.

The output of the INA was acquired by use of the data acquisition device NI USB-6009 (National Instruments, Austin, Texas, USA), which is connected to a laptop via USB. Signals were digitized with a sampling frequency of $12 \mathrm{kSamples}$.

c) Data processing: Further data processing was implemented in Matlab (MathWorks, Natick, Massachusetts, USA), version 2017b. The measured signal was band-pass filtered at the reference frequency $f_{\text {ref }}=1.15 \mathrm{kHz}$, demodulated and finally smoothed by a moving root mean square filter with a window width of 240 samples, i. e. $0.02 \mathrm{~s}$.

d) Patient's safety: The PCB is powered by the power bank, the data acquisition card by USB connection to the laptop. With this laptop running in battery mode during measurement, the entire system has no connection to mains power supply as required by the European norm EN 60601-1 for patient's safety.

\section{B. Measurement Protocol}

The electrodes under investigation were fixed on the left side of the chest, close to the heart, by an elastic tape, in order to simulate a setup where the electrodes are integrated into textiles (Fig. 2). Isolation between electrode and skin was provided by always the same cotton T-shirt. The study population comprised 13 male participants (23-29 years old).

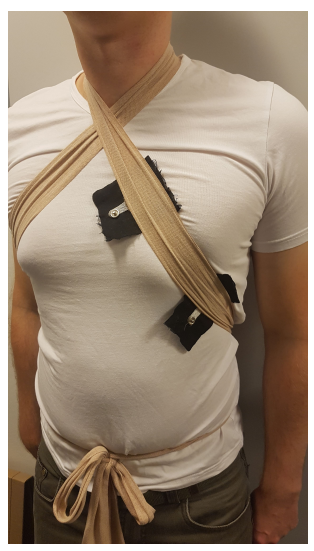

Fig. 2. Fixation of the electrodes to the chest by an elastic tape.

The study protocol comprised the following three phases:

1) Hands closed in front of the body; alternation of hands pressed together and relaxed. This leads to an alternating tension and relaxation of the chest muscles without body movement.

2) Movements of the left arm

3) Movements of the right arm

All phases were performed sitting on a chair. The movement phases consisted of $15 \mathrm{~s}$ in rest, $30 \mathrm{~s}$ performing the specified movement, $15 \mathrm{~s}$ in rest and another $20 \mathrm{~s}$ of movements. The protocol was repeated for each kind of electrode. When changing the electrode, the fixation tape remained on the body in order to ensure unchanged fixation.

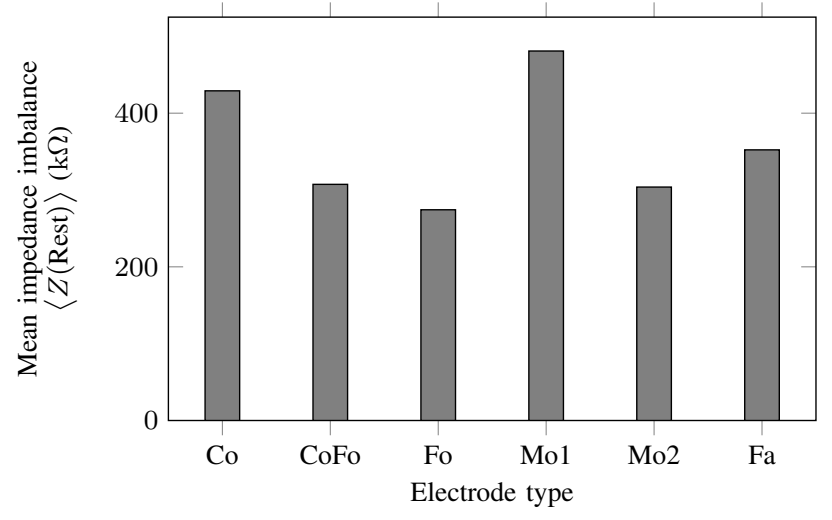

Fig. 3. Static impedance imbalance. 


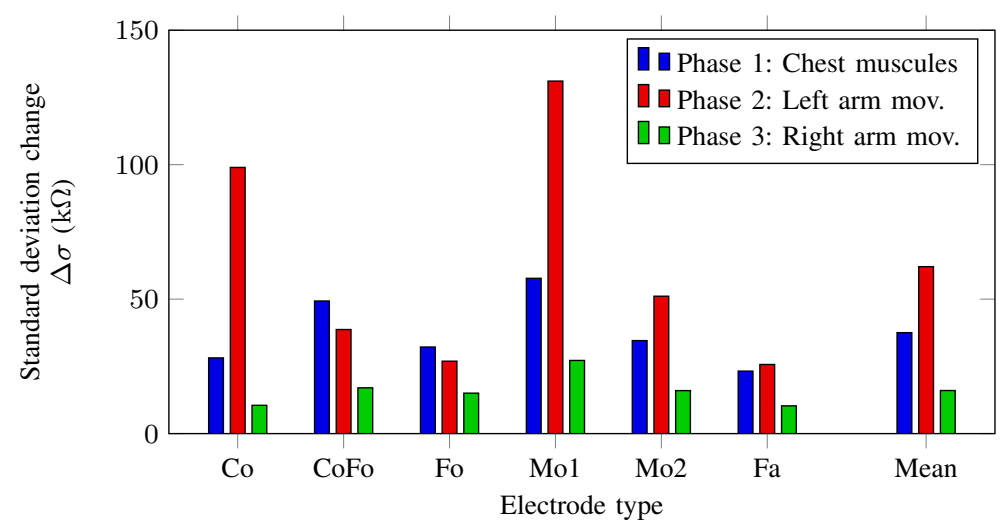

(a) Dynamic impedance imbalance, separately for each phase of study protocol and each electrode. Average over all electrodes on the right.

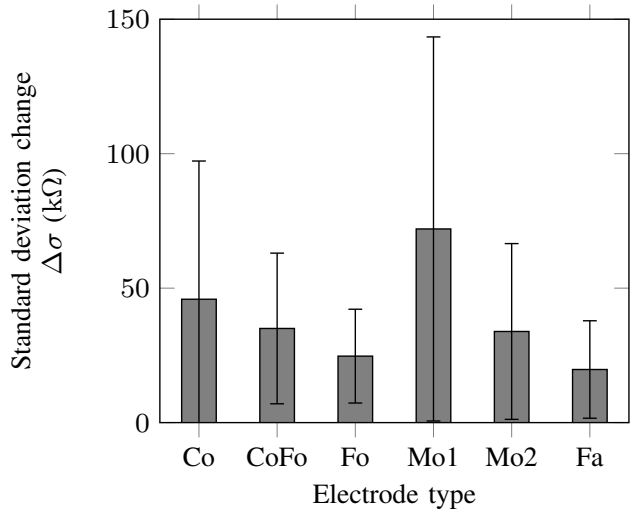

(b) Average over all phases of the study protocols. Error bars indicate inter-person variation.

Fig. 4. Dynamic impedance imbalance.

\section{Data Evaluation}

The imbalance between the contact impedances of the electrodes was quantified in the following two ways: As the fixation of the electrodes might lead to a static imbalance, the mean measured impedance imbalance at rest, $\langle Z($ Rest $)\rangle$, was computed. For the motion-induced, i. e., dynamic changes of the imbalance, the difference $\Delta \sigma$ between the standard deviation in phase $X(X=1,2,3)$ and the standard deviation at rest were used. In the latter case, standard deviation of $\Delta \sigma$ was obtained over all study subjects as an indicator of interperson variation.

\section{RESUlTS AND DiscUSSION}

Figures 3 and 4 show the static and dynamic impedance imbalances, respectively, for the various electrode types.

Static impedance imbalance varies between 270 and $480 \mathrm{k} \Omega$, with the highest values found in the moss and the copper plate electrodes. The performance of the former is improved by adding foam (imbalance reduced by $29 \%$ in CoFo compared to $\mathrm{Co}$ ). Also an increase in electrode area is found to decrease the imbalance, as can be seen when comparing Mo1 and Mo2 (reduction by $37 \%$ due to an increase of diameter by $50 \%$ ). The smallest impedance imbalance value is exhibited by the foam electrode, while the fabric electrode shows an intermediate performance.

The dynamic impedance imbalance is shown separately for each electrode type and each study protocol phase in Fig. 4(a); the average over all electrode types is given on the right side of the plot. This average behavior is found in all different types of electrodes except for one case ( $\mathrm{CoFo})$ : Phase 2, i. e., movement of the left arm has a strong effect on the nearby electrodes, whereas phase 3, i. e., movement of the right arm has the smallest impact on the contact impedance imbalance, even compared to chest muscle tension. This is explained by the sufficiently large distance between right arm and the electrodes attached close to the heart and a fixation that is hardly affected by movements of this arm (see also Fig. 2).
The overall performance of the electrode types is summarized in Fig. 4(b). The smallest sensitivity against movement artifacts is found in the foam and the fabric electrodes with mean standard deviation change of 25 and $20 \mathrm{k} \Omega$. Again, the moss embroidered electrode exhibits the largest $\Delta \sigma(72 \mathrm{k} \Omega)$, followed by the rigid copper plate electrode $(46 \mathrm{k} \Omega)$. As for the static imbalance, the addition of conductive foam and increase of the electrode area improves the performance.

The inter-person variation, as indicated by the error bars, approximately agrees with $\Delta \sigma$ itself. Hence, the lowest variation is found in the foam and fabric electrodes.

The rigid form of the pure copper electrode Co is assumed to be the main cause of its high tendency to contact impedance imbalances (see also the evaluation of an ECG system with these electrodes under body movements [17]): The electrode cannot adapt to the uneven body surface, such that gaps between electrode, T-shirt and skin result. Adding foam can partly compensate this effect but does not reach the flexibility that is provided by the pure foam and fabric electrodes. The moss, however, while being sufficiently flexible, is highly compressible, which is assumed to be responsible for the observed large variations in contact impedance and for the resulting sensitivity against motion artifacts.

\section{SUMmARY AND OUTLOOK}

The material of the electrodes for ECG capture has a strong impact both on the static contact impedance (im)balance and on its dynamic behavior induced by body movement. The best overall performance was found in the electrodes made of flexible and incompressible material, whereas the rigid and soft materials exhibited the highest tendency to imbalances.

Further research steps will include an investigation of contact impedance with larger study population and protocol as well as an exploration of approaches to compensate for imbalances by hardware means. Furthermore, sources of motion artifacts beyond contact impedance imbalance and potential countermeasures have to be surveyed in order to realize robust capacitive ECG system applicable for daily use. 


\section{REFERENCES}

[1] J. M. Engel, V. Mehta, R. Fogoros, and A. Chavan, "Study of arrhythmia prevalence in nuvant mobile cardiac telemetry system patients," in Proc. 2012 Ann. Int. Conf. IEEE Eng. Med. Biol. Soc. IEEE, 2012, pp. 2440 2443.

[2] S. S. Lobodzinski, "ECG patch monitors for assessment of cardiac rhythm abnormalities," Prog. Cardiovasc. Dis., vol. 56, no. 2, pp. 224 229, 2013.

[3] A. M. Chan, N. Selvaraj, N. Ferdosi, and R. Narasimhan, "Wireless patch sensor for remote monitoring of heart rate, respiration, activity, and falls," in Proc. 2013 35th Ann. Int. Conf. IEEE Eng. Med. Biol. Soc. IEEE, 2013, pp. 6115-6118.

[4] S. S. Lobodzinski and M. M. Laks, "New devices for very long-term ECG monitoring," Cardiol. J., vol. 19, no. 2, pp. 210-214, 2012.

[5] E. Fung, M.-R. Järvelin, R. N. Doshi, J. S. Shinbane, S. K. Carlson, L. P. Grazette, P. M. Chang, R. S. Sangha, H. V. Huikuri, and N. S. Peters, "Electrocardiographic patch devices and contemporary wireless cardiac monitoring," Front. Physiol., vol. 6, 2015.

[6] P. M. Barrett, R. Komatireddy, S. Haaser, S. Topol, J. Sheard, J. Encinas, A. J. Fought, and E. J. Topol, "Comparison of 24-hour holter monitoring with 14-day novel adhesive patch electrocardiographic monitoring," Am. J. Med., vol. 127, no. 1, pp. 95-e11, 2014.

[7] M. A. Rosenberg, M. Samuel, A. Thosani, and P. J. Zimetbaum, "Use of a noninvasive continuous monitoring device in the management of atrial fibrillation: a pilot study," Pacing Clin. Electrophysiol., vol. 36, no. 3, pp. 328-333, 2013

[8] M. P. Turakhia, D. D. Hoang, P. Zimetbaum, J. D. Miller, V. F. Froelicher, U. N. Kumar, X. Xu, F. Yang, and P. A. Heidenreich, "Diagnostic utility of a novel leadless arrhythmia monitoring device," Am. J. Cardiol., vol. 112, no. 4, pp. 520-524, 2013.
[9] E. Nemati, M. J. Deen, and T. Mondal, "A wireless wearable ECG sensor for long-term applications," IEEE Commun. Mag., vol. 50, no. 1, pp. 36-43, 2012.

[10] Y. Sun and X. B. Yu, "Capacitive biopotential measurement for electrophysiological signal acquisition: A review," IEEE Sens. J., vol. 16, no. 9, pp. 2832-2853, 2016.

[11] S. Heuer, "Ambiente kapazitive EKG-Messung: Elektroden, Systeme und Konzepte," Ph.D. dissertation, Karlsruhe, Karlsruher Institut für Technologie (KIT), 2011.

[12] J. Kirchner, N. Roth, A. Meyer, and G. Fischer, "ECG measurement by use of passive capacitively coupled electrodes," in Proc. 2016 IEEE Sensors, 2016, pp. 1-3.

[13] V. X. Afonso, W. J. Tompkins, T. Q. Nguyen, K. Michler, and Shen Luo, "Comparing stress ecg enhancement algorithms," IEEE Engineering in Medicine and Biology Magazine, vol. 15, no. 3, pp. 37-44, May 1996.

[14] P. S. Hamilton, M. G. Curley, R. M. Aimi, and C. Sae-Hau, "Comparison of methods for adaptive removal of motion artifact," in Computers in Cardiology 2000. Vol.27 (Cat. 00CH37163), Sep. 2000, pp. 383-386.

[15] T. Degen and T. Loeliger, "An improved method to continuously monitor the electrode-skin impedance during bioelectric measurements," in 2007 29th Annual International Conference of the IEEE Engineering in Medicine and Biology Society, 2007, pp. 6294-6297.

[16] LT1007/LT1037. Low Noise, High Speed Precision Operational Amplifiers, Linear Technology Corp., IT/CPI 1101 1.5K Rev. B.

[17] J. Kirchner, S. Pfeiffer, and G. Fischer, "Passive capacitive ECG sensing: Assessment of signal quality during different types of body movement," in 2018 IEEE International Symposium on Medical Measurements and Applications (MeMeA), June 2018, pp. 1-5. 